\title{
Ventajas del ejercicio físico de bajo impacto para la atención en trastornos del sueño en adultos de 60 años y más de edad no institucionalizados
}

Advantages of low impact physical exercise for attention in sleep disorders in noninstitutionalized adults over 60 years of age

Natalia Arias Arias ${ }^{1} \square$, Evelyn Marín Rivera ${ }^{2}$

\section{Resumen}

Filiación:

1. Trabajadora independiente. Alajuela, Costa Rica.

2. Hospital Nacional de Geriatría y Gerontología. Caja Costarricense del Seguro Social. San Jose, Costa Rica.

Correspondencia: $\square$ Natalia Arias Arias.

email: natiarias10@gmail.com

- - - - - - - - - -

Abreviaturas: AM: Adulto(s) Mayor(es)

MOR: Movimientos oculares rápidos

NOMOR: Sin movimientos oculares rápidos

Forma de citar: Arias Arias N, Marín Rivera E. Ventajas del ejercicio físico de bajo impacto para la atención en trastornos del sueño en adultos de 60 años y más de edad no institucionalizados. Rev Ter. 2018;12(2):23-33.

Financiamiento:

Autofinanciado

Conflictos de Interés:

El autor declara no tener conflictos de interés en la presente publicación
El adulto mayor (AM) pierde con los años, su capacidad para conseguir un sueño que sea continuado y consolidado. La intervención no farmacológica de los trastornos del sueño, ha demostrado beneficios para la producción de cambios en los hábitos del sueño; dentro de este abordaje se destacan el control de estímulos, la restricción del sueño y las técnicas de relajación, las cuales reducen limitaciones como el costo económico, el tiempo, la falta de profesionales para la preparación farmacológica y además reducen los índices de polifarmacia característicos del adulto mayor. Con el objetivo de obtener evidencia sobre las ventajas que genera el ejercicio de bajo impacto en el AM no institucionalizado con trastornos del sueño, se realizó una revisión narrativa.

Artículos científicos encontrados en Pubmed, Elsevier y Google Académico, publicados en inglés y español del año 2010 al 2016. Utilizando los descriptores ejercicio físico, adultos mayores, institucionalización y trastornos del sueño, se seleccionaron los artículos con evidencia existente, sobre las ventajas del ejercicio físico de bajo impacto en los trastornos del sueño en los adultos de 60 años y más de edad. La evidencia encontrada demuestra que los ejercicios de bajo impacto como la caminata, el pedaleo en bicicleta estática, la natación, la hidrogimnasia, el subir y bajar escaleras, el baile, el yoga y la gimnasia aeróbica de bajo impacto, son efectivos para aumentar la capacidad de realizar actividades de la vida cotidiana y para disminuir el consumo de medicamentos.

Conclusiones: Los efectos del ejercicio físico de bajo impacto van más allá del conocido beneficio cardiovascular, debido a que se van a presentar por su parte respuestas positivas en la calidad del sueño y disminución de los trastornos del sueño presentes en el adulto mayor.

Descriptores: Ejercicio físico, adultos mayores, sueño, trastornos del sueño. 


\section{Abstract}

The older adult loses their ability to achieve continuous and consolidated rest over the years. Non-pharmacological intervention of sleep disorders have shown benefits for the production of changes in sleep habits; within this approach, stimulus control, sleep restriction and relaxation techniques are highlighted, which reduce limitations such as economic cost, time, lack of professionals for pharmacological preparation and also reduce the polypharmacy rates characteristic of the elderly.

In order to obtain evidence on the advantages that low-impact exercise generates in the noninstitutionalized older adult with sleep disorders, a narrative review of the scientific articles found in Pubmed, El Sevier and Google Scholar, published in English and Spanish was conducted from 2010 to 2016. Using the descriptors physical exercise, older adults, institutionalization and sleep disorders, articles with existing evidence were selected, about the advantages of low impact physical exercise on sleep disorders in adults aged 60 years and over.

The evidence found shows that low-impact exercises like walking, cycling, swimming, hydrogymnastics, climbing and descending stairs, dancing, yoga and low impact aerobic gymnastics are effective in increasing the ability to perform activities of daily living and to decrease drug use.

Conclusions: The effects of low-impact physical exercise go beyond the well-known cardiovascular benefit, due to other hand positive responses in the quality of sleep and decrease of sleep disorders present in the elderly.

Keywords: Physical exercise, elderly, sleep, sleep disorders.

\section{Introducción}

El envejecimiento es una disminución progresiva de las funciones de los diferentes órganos y sistemas vitales, para responder a cambios en el medio interno y en el medio ambiente. En Costa Rica, la persona adulta mayor es considerada como tal a partir de los 65 años de edad ${ }^{1}$.

Según datos de la Organización Mundial de la Salud (OMS) establecidos en el 2012, entre los años 2000 y 2050 la cantidad de personas mayores de 60 años, se duplicará, pasando del $11 \%$ al $22 \%$; es decir para el 2020 habrá 20 millones adultos mayores².

En Costa Rica, según datos del Instituto Nacional de Estadística y Censo (INEC), para el último censo registrado durante el año 2011, la población adulta mayor de más de 65 años corresponde a 144830 hombres y 166882 mujeres para un total de 311712 personas $^{3}$.

Las proyecciones nacionales de población adulta mayor para el 2015, 2025, 2035 y 2050 corresponden a $7,3 \%, 9 \%, 15 \%$ y $21 \%$ respectivamente. Esto en números absolutos significa pasar en el año 2015 de 355637 AM a 1262310 en el año 20504,5.

En la actualidad el sueño es considerado como un proceso fisiológico de gran importancia, siendo indispensable en las diversas etapas de la vida. Se considera una condición de desconexión con el entorno, que se caracteriza por ser un fenómeno cíclico, natural y fácilmente reversible mediante estímulos que se definen por el comportamiento de la persona mientras duerme ${ }^{6,7,8}$. Existen datos que establecen que el $10 \%$ de las personas sufren insomnio que altera su calidad de vida y $3-4 \%$ presenta apneas durante el sueño ${ }^{6}$.

En el AM los trastornos del sueño hoy en día, son el principal problema en cuanto a la incapacidad para mantener el sueño durante toda la noche, conciliarlo al acostarse y además convivir con los constantes despertares nocturnos ${ }^{6}$. Los AM con trastornos del sueño normalmente son tratados únicamente con 
fármacos, los cuales crean dependencia, aumentan la tasa de polifarmacia y por ende sus efectos secundarios. Por esta razón, es imprescindible conocer alternativas no farmacológicas para abordar los trastornos del sueño en la población adulta mayor, enfocándose en el ejercicio físico, que por su parte ha demostrado beneficios en el sistema musculo esquelético, área cognitivo-conductual, disminución de caídas, manejo de enfermedades crónicas, aumento de la calidad de vida, entre otros $^{9-12}$.

Se consideró no incluir los AM institucionalizados pues éstas presentan diferentes características relacionadas con un perfil de aislamiento, inactividad y fragilidad. Existe una fuerte asociación entre los cambios a nivel funcional de los AM y el riesgo de caídas o mortalidad dentro del proceso de institución ${ }^{13}$ además de presentar una menor realización de ejercicio físico ${ }^{14}$. Según la evidencia, las personas institucionalizadas sufren un mayor número de caídas que las personas que viven en la comunidad $^{13,15}$.

El objetivo de esta revisión fue describir la evidencia publicada en la literatura, sobre las ventajas que genera el ejercicio físico de bajo impacto en el adulto de 60 años y más no institucionalizado con trastornos del sueño.

\section{Materiales y métodos}

La investigación que se realizó es un estudio descriptivo, de revisión narrativa de la evidencia existente donde se describen los beneficios del ejercicio físico de bajo impacto en los trastornos del sueño del AM no institucionalizado.

Para la búsqueda bibliográfica se utilizaron las siguientes bases de datos: Pubmed, Elsevier y Google Académico. Considerando los descriptores ejercicio físico, adultos mayores, institucionalización y trastornos del sueño se seleccionaron aquellos artículos científicos, de revisión bibliográfica, ensayos clínicos, estudios de cohorte y casos y controles, publicados durante el periodo 2010 2016, tanto en inglés como español.

Se incluyeron un total de 35 artículo, 5 ensayos clínicos controlados y uno no controlado, una revisión sistemática, 5 estudios transversales analíticos (uno de ellos de base poblacional) y 23 revisiones de calidad.

\section{Resultados y Discusión}

Varela y colaboradores ${ }^{16}$, mencionan en las Guías de Atención Primaria de la Organización Panamericana de la Salud (OPS), que un sueño nocturno adecuado permite mantener una vida activa y saludable en el adulto mayor.

El comportamiento del sueño debe ser importante durante el envejecimiento, no solamente en cantidad de horas, sino en la calidad y continuidad de éste. En esta etapa el AM presenta cambios en la macro y microestructura del sueño ${ }^{17}$.

La recomendación en cuanto a la duración del sueño es de 7 a 8 horas diarias en personas mayores de 65 años. Si a lo largo de esta etapa, el adulto logra conciliar un sueño reparador durante esta cantidad de horas, va a presentar un mejor funcionamiento cognitivo, una menor cantidad de enfermedades físicas y mentales y además una mejor calidad de vida $^{17}$.

\section{Trastornos del sueño más frecuentes en la población de adultos de 60 años y más.}

El AM pierde con los años, su capacidad para conseguir un sueño que sea continuado y consolidado, lo que va a provocar que se distribuyan sus horas de sueño durante las 24 horas del día y que aumente la frecuencia de las siestas diurnas. Además acompañado de varios factores causales como la disminución de la melatonina nocturna, 
la aparición de diferentes enfermedades, la polifarmacia y los aspectos psicosociales ${ }^{17,18,19-22}$.
Los trastornos del sueño más frecuentes y de mayor importancia, se encuentran en el cuadro 1.

\section{Cuadro 1. Trastornos del sueño más frecuentes en el adulto mayor.}

\begin{tabular}{|c|c|c|}
\hline Trastorno del sueño & Definición & Características \\
\hline Insomnio & $\begin{array}{l}\text { Alteración del sueño que se relacio- } \\
\text { na con somnolencia diurna, fatiga, } \\
\text { disfunción cognitiva, caídas, depre- } \\
\text { sión, aumento de la mortalidad y } \\
\text { disminución en la calidad de vida }{ }^{14} \text {. }\end{array}$ & $\begin{array}{l}\text { Deterioro social, laboral, educativo, académi- } \\
\text { co, del comportamiento y funcionamiento de } \\
\text { la persona }{ }^{20-22} \text {. }\end{array}$ \\
\hline $\begin{array}{l}\text { Trastornos relaciona- } \\
\text { dos con la respiración }\end{array}$ & $\begin{array}{l}\text { Disminución o colapso temporal de } \\
\text { las vías aéreas, que producen un } \\
\text { sueño más superficial y despertares } \\
\text { frecuentes, llevando a una somno- } \\
\text { lencia diurna }{ }^{23} \text {. }\end{array}$ & $\begin{array}{l}\text { Prevalencia de un } 2 \% \text { en mujeres y } 4 \% \text { en } \\
\text { hombres. Se asocia con la obesidad, la hiper- } \\
\text { tensión pulmonar y sistémica, arritmias car- } \\
\text { díacas y mayor mortalidad }{ }^{24} \text {. }\end{array}$ \\
\hline $\begin{array}{l}\text { Trastornos del ritmo } \\
\text { circadiano }\end{array}$ & $\begin{array}{l}\text { Somnolencia excesiva, insomnio o } \\
\text { ambos, debido a la interrupción que } \\
\text { se ocasiona en el sueño }\end{array}$ & $\begin{array}{l}\text { Deterioros sociales o laborales y alteración } \\
\text { del ciclo normal de sueño-vigilia }{ }^{21}\end{array}$ \\
\hline $\begin{array}{l}\text { Trastornos inducidos } \\
\text { por sustancias o medi- } \\
\text { camentos }\end{array}$ & $\begin{array}{l}\text { Fármacos que pueden provocar o } \\
\text { exacerbar el insomnio en el adulto } \\
\text { mayor }\end{array}$ & $\begin{array}{l}\text { Cualquier droga o fármaco que altera la cali- } \\
\text { dad o la arquitectura del sueño }{ }^{25}\end{array}$ \\
\hline
\end{tabular}

Elaboración de los autores. Fuente: Martínez $\mathrm{M}^{17}$, López $\mathrm{F}$ et $\mathrm{al}^{23}$, Arlington $\mathrm{V}^{24}$, Ganado $\mathrm{E}$ et al ${ }^{25}$, Flores $\mathrm{A}$ et $\mathrm{al}^{26}$, Fernández $\mathrm{A}^{27}$, Fuentes $\mathrm{S}$ et $\mathrm{al}^{28}$.

\section{Efectos del ejercicio físico de bajo impacto en trastornos del sueño de los adultos mayores}

La ejecución de al menos 30 minutos de ejercicio moderado, por 5 días a la semana, reduce el riesgo de enfermedades comunes no trasmisibles. Se debe tomar en cuenta que este tipo de enfermedades requieren de normas de ejercicio específicas, las cuales deber individualizarse y ser adecuadamente prescritas por un profesional ${ }^{29,} 30$.

Los ejercicios de bajo impacto se recomiendan más para la población adulta mayor siempre y cuando posean una adecuada prescripción. Las actividades aeróbicas como la caminata, el ciclismo o pedaleo en bicicleta estática, la natación, la hidrogimnasia, el subir y bajar escaleras, el baile, el yoga y la gimnasia aeróbica de bajo impacto son las actividades preferibles sobre otras que produzcan un alto impacto sobre el organismo, debido a la alta incidencia de lesiones en las personas de la tercera edad $^{31}$. El cuadro 2 resume las características y beneficios de las intervenciones de bajo impacto en los trastornos del sueño en adultos mayores 
Cuadro 2. Características y beneficios de los ejercicios físicos de bajo impacto en los trastornos del sueño en adultos mayores.

\begin{tabular}{|c|c|c|}
\hline Intervención & Características & Beneficios \\
\hline Caminatas $^{32-33}$ & $\begin{array}{l}\text { Ideal combinarlo con ejerci- } \\
\text { cios de fuerza y flexibilidad. } \\
\text { Sesiones de } 70 \text { minutos. }\end{array}$ & $\begin{array}{l}\text { Aumento la habilidad para realizar actividades de la } \\
\qquad \text { vida diaria. } \\
\text { Mejora la capacidad aeróbica. }\end{array}$ \\
\hline Gerontogimnasia $^{9}$ & $\begin{array}{l}\text { Tiene un mayor efecto sobre } \\
\text { la calidad del sueño. }\end{array}$ & $\begin{array}{l}\text { Reducción importante del consumo farmacológico } \\
\text { para dormir. } \\
\text { Baja los niveles de hipersomnio. } \\
\text { Reducción del insomnio. }\end{array}$ \\
\hline Aquagym $^{9}$ & $\begin{array}{l}\text { Efecto combinado con las } \\
\text { cualidades del agua. }\end{array}$ & $\begin{array}{l}\text { Efecto medio sobre la satisfacción subjetiva del sue- } \\
\text { ño y reducción del hipersomnio. }\end{array}$ \\
\hline Pilates $^{9}$ & $\begin{array}{l}\text { Realización bajo supervisión } \\
\text { profesional. }\end{array}$ & $\begin{array}{l}\text { Efecto medio sobre la satisfacción subjetiva del sue- } \\
\text { ño. }\end{array}$ \\
\hline $\begin{array}{l}\text { Técnicas de relaja- } \\
\text { ción }^{9}\end{array}$ & $\begin{array}{l}\text { Efecto combinado con la res- } \\
\text { piración. }\end{array}$ & $\begin{array}{c}\text { Fuerte efecto sobre el hipersomnio } \\
\text { Efecto medio sobre el insomnio } \\
\text { Reducción del consumo farmacológico para dormir } \\
\text { Efecto algo más bajo sobre la satisfacción subjetiva } \\
\text { del sueño. }\end{array}$ \\
\hline $\begin{array}{l}\text { Caminatas de mínimo } \\
200 \text { minutos por se- } \\
\text { mana }^{34}\end{array}$ & $\begin{array}{l}\text { Grado de esfuerzo percibido } \\
\text { entre } 13 \text { y } 15 \text { durante al me- } \\
\text { nos } 30 \text { minutos en la Escala } \\
\text { de Borg. }\end{array}$ & $\begin{array}{l}\text { Cambios significativos en la movilidad, salud y auto- } \\
\qquad \text { valoración } \\
\text { Impacto positivo en la somnolencia diurna, produc- } \\
\text { tividad social, actividad, vigilia e intimidad. }\end{array}$ \\
\hline Natación adaptada ${ }^{35}$ & $\begin{array}{l}\text { Sujetos con falta de autono- } \\
\text { mía y con problemas de de- } \\
\text { pendencia }\end{array}$ & $\begin{array}{l}\text { Mejora aspectos psíquicos, físicos y fisiológicos; ma- } \\
\text { yor bienestar y autonomía. } \\
\text { Frena el deterioro propio del envejecimiento. }\end{array}$ \\
\hline Terapia de baile ${ }^{11}$ & $\begin{array}{l}10 \text { minutos de movilidad y } \\
\text { ejercicios de baja intensidad, } \\
20 \text { minutos de movimientos } \\
\text { más activos dentro del baile, } \\
10 \text { minutos de coreografía y } \\
\text { relajación de } 10 \text { minutos }\end{array}$ & $\begin{array}{l}\text { Calidad del sueño mejoró con respecto a la duración } \\
\qquad y \text { toma de medicación para dormir } \\
\text { Eficacia del sueño mejoró de un } 85,15 \% \text { a un } 90 \%\end{array}$ \\
\hline
\end{tabular}

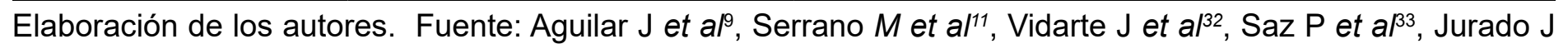
et $\mathrm{a}^{{ }^{34}}$, Gallejo J et aß ${ }^{\beta 5}$. 
Dentro de los resultados encontrados se destaca el estudio intervencional de Vidarte et $a^{\beta 2}$, quienes realizaron una investigación con 38 AM de 60 años con un grupo control y otro experimental, el cual fue sometido a un programa de ejercicio físico por 12 semanas, basadas en actividades de locomoción específicamente caminatas, fuerza y flexibilidad. En el estudio se concluye que el ejercicio físico es una estrategia que aumenta la habilidad de los AM para realizar sus actividades de la vida diaria y además previene la disminución en el acondicionamiento cardiorrespiratorio característico de la edad ${ }^{32}$, 33. Además, la capacidad aeróbica se incrementó significativemente en un $10-25 \%$ lo cual ayuda a mejorar las condiciones de la persona en cuanto a la realización de sus actividades con respecto al grupo control. En referencia al aumento de la autoconfianza de las personas para caminar de forma segura luego del programa de ejercicios, se determina la presencia de una mayor energía y resistencia para realizar sus actividades cotidianas y la participación en el entorno ${ }^{32}$.

Otro estudio realizado por Aguilar et al ${ }^{9}$ en el 2015, sobre la aplicación de programas de actividad física en la calidad del sueño en 580 personas mayores de 55 años, durante un período de 9 meses con 3 sesiones por semana y de una hora de duración y que incluía: gerontogimnasia, aquagym, Pilates y técnicas de relajación. Se confirma que el programa de gerontogimnasia, fue el que tuvo un mayor efecto sobre la calidad del sueño, generando un fuerte impacto sobre la reducción del consumo farmacológico en un 19\% y de los niveles de hipersomnio. También se aportó un efecto medio sobre la reducción del insomnio con una media pre-test de 20,79 comparada a post-test de $17,41(p=0,001)$ y un efecto algo más bajo en la satisfacción subjetiva de la calidad del sueño con una media de 4,27 pre-test y 4,69 posttest $(p=0,022)$. El programa de Pilates, solo tuvo un efecto medio sobre la satisfacción subjetiva del sueño con una media pre-test de 4,24 y post-test de 5,14 $(p=0,019)$. $Y$ en cuanto al de aquagym, se da un efecto medio sobre la satisfacción subjetiva y una reducción del hipersomnio.

Por último el programa de relajación presentó un fuerte efecto sobre el hipersomnio, un efecto medio sobre el insomnio, una reducción del consumo farmacológico y un efecto algo más bajo sobre la satisfacción subjetiva del sueño. Dentro de esta misma investigación se demuestra que la práctica de ejercicio físico regular, específicamente aeróbico, activa las principales estructuras cognoscitivas de los individuos, actuando como elemento de prevención de trastornos relacionados con el sueño. Uno de los principales hallazgos, fue que no se encontraron diferencias estadísticamente significativas en cuanto a sexo o edad en los beneficios de la actividad física sobre la calidad del sueño, esto debido a que las alteraciones del sueño se asocian más a factores relacionados con el estilo de vida psicológico, fisiológico y sanitario. Los resultados confirman la hipótesis, debido a que el grupo experimental obtuvo una reducción significativa en variables de insomnio, hiperinsomnio y reducción de fármacos para dormir, con respecto al grupo control.

Por su parte, un ensayo clínico controlado realizado por Jurado et $a^{\beta 4}$, en 23 adultos mayores, donde la intervención consistió en caminatas de al menos 200 minutos por semana, durante varias sesiones en 6 meses, siempre manteniendo un ritmo rápido en cada una de ellas para producir un grado de esfuerzo percibido entre 13 y 15 durante al menos 30 minutos en la Escala de Borg. Dentro de los efectos importantes que se observaron en este ensayo clínico basado en un diario de caminatas, se destacan en el grupo de intervención, cambios significativos en la movilidad, la salud, la autovaloración $(p<0,05)$ y las actividades cotidianas, la ansiedad y la depresión mejoraron con el ejercicio 
pero sin lograr significación estadística. Además, se presentó un impacto de la somnolencia diurna en el estado funcional de los pacientes que realizaron ejercicio, así como en la productividad social, actividad diurna, vigilia e intimidad $(p<0,05)$. Se concluye que en los pacientes con Síndrome de apneas-hipoapneas leve analizados en el grupo experimental, el ejercicio realizado se asocia a una mejor calidad de vida y a un impacto percibido por la somnolencia diurna.

Gallego et ${ }^{/ 35}$ realizaron un programa de intervención sobre natación adaptada para 33 personas mayores, hombres y mujeres con falta de autonomía y con problemas de dependencia, realizado durante ocho meses, dos veces por semana y por un período de 45 minutos, confirma que el ejercicio físico adaptado, mejora aspectos psíquicos, físicos y fisiológicos, proporcionando mayor bienestar y autonomía en la persona que lo realiza y a la vez frena el deterioro propio del envejecimiento. Además se determinó que los hombres $(p<0,09)$ presentan una autoestima más alta que las mujeres $(p<0,499)$.

En un estudio realizado por Subirats et a ${ }^{10}{ }^{10}$ en el año 2010, donde se presentan una serie de revisiones sistemáticas en torno a la prevención de la mortalidad por medio de la prescripción del ejercicio; se incluye una en particular que sometió a 19842 hombres a ejercicio físico y concluyó que cualquier forma de actividad física diaria reduce $41 \%$ de riesgo de padecer trastornos psicológicos, destacando la reducción tanto de síntomas de ansiedad como de depresión, con una prescripción de actividad física de 20 minutos semanales. Además de realizar ejercicio aeróbico, el complemento con ejercicio de fuerza con pesas, también produjo una mejoría del sueño, la fuerza, la calidad de vida y la depresión. En relación con el insomnio, la práctica de ejercicio por la mañana mejora la calidad del sueño en la población sedentaria, con obesidad, mujeres posmenopáusicas, AM y cuidadores de pacientes con demencia.

Por otra parte, Serrano et $a^{11}$ en el año 2016, realizaron un estudio controlado aleatorizado con un grupo control de 32 participantes y otro grupo de 35 personas. Probaron un programa de terapia de baile donde las sesiones incluían 10 minutos de movilidad y de ejercicios de baja intensidad, 20 minutos de movimientos más activos dentro del baile, 10 minutos de coreografía y una relajación de 10 minutos, para la mejora de parámetros cardiovasculares y del sueño, en mujeres entre los 65 y 75 años. Dicho programa de 8 semanas aportó diferentes efectos positivos tales como: incremento del equilibrio, reducción de caídas, beneficios cardiovasculares, agilidad, flexibilidad, el descanso nocturno y el alivio de la depresión. La calidad del sueño mejoró considerablemente al final del programa de terapia de baile con respecto a la duración, eficacia y toma de medicación $(p<0,05)$. La eficacia del sueño mejoró de un $85,15 \%$ a un $90 \%$ con la terapia de baile, en cambio en el grupo control el cambio fue solamente de un $0,43 \%$. Además se concluye que los beneficios adicionales del baile como una forma agradable de ejercicio de bajo impacto que promueve la interacción social y mejora su asistencia regular, es una práctica rentable, no costoso, no aburrida y que por último se pueda introducir fácilmente en la comunidad.

Karimi et $a l^{12}$, comentan que ante la ausencia de práctica de ejercicio físico en las personas adultas mayores, existe un aumento de riesgo de padecer osteoporosis, obesidad, depresión, enfermedades cardiovasculares, diabetes tipo 2, cáncer de colon y trastornos del sueño. Ellos realizaron un estudio durante 8 semanas donde se probó un programa de ejercicio físico dirigido a un grupo seleccionado de 46 hombres de edad avanzada, el cual se caracterizó por un calentamiento y fases de preparación de 5 minutos, una caminata lo más rápida posible por 10 minutos, una caminata lenta para el enfriamiento de 
5 minutos y por último el descanso y la relajación de 10 minutos. Los autores reportaron los siguientes resultados del grupo experimental, el aumento en la calidad de sueño $(p=0,001)$ en los AM junto con la calidad de sueño mental $(p=0,01)$, una reducción en el tiempo que se necesita para dormirse $(p=0,001)$, un incremento en el término del sueño $(p=0,004)$, una disminución en la necesidad de utilizar medicamentos para dormir $(p=0,09)$ y una reducción en los problemas para realizar las actividades diarias $(p=0,02)$. Lo que confirma, que la utilización del ejercicio físico como intervención no farmacológica, es una de las herramientas significativas para tratar los trastornos del sueño ${ }^{12}$.

Las ventajas que se obtienen a raíz de la realización de ejercicio físico de bajo impacto en los AM no institucionalizados de 60 años y más, se van a confirmar según los datos anteriormente presentados; resaltando la realización de los ejercicios guiados por un profesional, con una correcta prescripción y en los horarios adecuados, generando efectos positivos funcionales, mejorando las diferentes zonas del cuerpo, la marcha, la autonomía, entre otras y por su parte en el área emocional y social, creando la motivación para la práctica del ejercicio y como ventaja fundamental, la reestructuración o mejoría en cuanto al sueño, disminuyendo los síntomas que afectan al AM en su calidad de vida.

\section{Conclusiones}

Los efectos del ejercicio físico de bajo impacto van más allá del conocido beneficio cardiovascular, debido a que se van a presentar múltiples mejorías en el sistema musculo esquelético, demás sistemas orgánicos, área cognitivo-conductual, disminución de caídas, manejo de enfermedades crónicas y por su parte respuestas positivas en la calidad del sueño y disminución de los trastornos del sueño presentes en el adulto mayor.
La adecuada prescripción del ejercicio influye en la mejoría de los trastornos del sueño; además se confirma una reducción significativa de la utilización de fármacos por parte de los AM que utilizan estas sustancias $u$ otros medios de apoyo para lograr conciliar el sueño. Lo anterior, corrobora una reducción de los índices de polifarmacia, principalmente durante la realización de gerontogimnasia y técnicas de relajación.

Los ejercicios físicos más recomendados para la población adulta mayor van a ser los de bajo impacto, gracias a los grandes beneficios que éstos aportan al cuerpo y a la mente. Entre los más reconocidos por sus efectos positivos, se destacan: la caminata, el ciclismo o pedaleo en bicicleta estática, la natación, la hidrogimnasia, el subir y bajar escaleras, el baile, el yoga y la gerontogimnasia; siempre contando con la supervisión de un especialista para la prevención de lesiones.

\section{Referencias}

1. González M. Trastornos del sueño en el adulto mayor. En: Morales Martínez F, compilador. Tratado de Geriatría y Gerontología. 2a ed. San José, CR: EDNASS; 2017. 655-662.

2. Benavides Rodríguez CL, García JA, Ariza Jiménez JF. El adulto mayor, caracterización funcional, nivel de actividad física y de fuerza: una revisión sistemática. En: $6^{\circ}$ Seminario Internacional en Actividad Física y Salud. Medellín: Universidad de Antioquía; 2015. 60-65. Disponible en: goo.gl/2FMzNS

3. Instituto Nacional de Estadística y Censo (CR). $X$ Censo Nacional de Población y VI de Vivienda: Resultados Generales [Internet]. San José, CR: INEC; 2011. Disponible en: http://www.inec.go.cr/ sites/default/files/documentos/inec_institucional/ estadisticas/resultados/repoblaccenso2011-15. pdf.pdf

4. Fondo de Población de Naciones Unidas. En- 
vejecimiento en el siglo XXI: Una celebración y un desafío. Resumen ejecutivo [Internet]. Nueva York: UNFPA; 2012. Disponible en: https://www. unfpa.org/sites/default/files/pub-pdf/Ageing\%20 Report\%20Executive\%20Summary\%20SPANISH\%20Final_0.pdf

5. Instituto Nacional de Estadística y Censos (CR). Estimaciones y Proyecciones de Población por sexo y edad 1950-2050 [Internet]. San José, CR: Centro Centroamericano de Población; 2013. Disponible en: http://www.inec.go.cr/sites/default/ files/documentos/poblacion/estimaciones_y_ proyecciones_de_poblacion/publicaciones/repoblacev1950-2050-01.pdf

6. Urrestarazu E, Escobar F, Iriarte J. El sueño y su patología. Medicine [Internet]. 2015;11(73):43854394. Doi: 10.1016/j.med.2015.02.004

7. Durán $\mathrm{S}$, Mattar $\mathrm{P}$, Bravo N, Moreno $\mathrm{C}$, Reyes S. Asociación entre calidad de vida y cantidad de sueño en adultos mayores de la Región Metropolitana y Región de Valparaíso, Chile. Rev Med Chile [Internet]. 2014;142(11):1371-1376. Disponible en: https://scielo.conicyt.cl/pdf/rmc/ v142n11/art02.pdf

8. Carrillo Mora P, Ramírez Peris J, Magaña Vázquez K. Neurobiología del sueño y su importancia: antología para el estudiante universitario. Rev Fac Med (Mex) [Internet]. 2013;56(4):5-14. Disponible en: http://www.scielo.org.mx/pdf/fac$\mathrm{med} / \mathrm{v} 56 \mathrm{n} 4 / \mathrm{v} 56 \mathrm{n} 4 \mathrm{a} 2 . \mathrm{pdf}$

9. Aguilar JM, Gallego J, Fernández JM, Pérez ER, Trigueros R, Alías A et al. Influencia de programas de actividad física en la calidad del sueño de personas mayores de 55 años. Rev Psicol Dep [Internet]. 2015;24(2):289-295. Disponible en: https://ddd.uab.cat/pub/revpsidep/revpsidep_ a2015v24n2/revpsidep_a2015v24n2p289.pdf

10. Subirats E, Subirats G, Soteras I. Prescripción del ejercicio: indicaciones, posología y efectos adversos. Med Clin (Barc) [Internet]. 2012;138(1):1824. Disponible en: http://handbook.usfx.bo/nue-
va/vicerrectorado/citas/SALUD_10/Medicina/33. pdf

11. Serrano M, Valenza CM, Serrano C, Aguilar E, Valenza G, Villaverde C. Efectos de un programa de terapia de baile en la calidad de vida, el sueño y la presión arterial en mujeres de media edad: un ensayo controlado aleatorizado. Med Clin (Barc) [Internet]. 2016;147(8):334- 339. Doi: 10.1016/j. medcli.2016.06.030

12. Karimi S, Soroush A, Towhidi F, Makhsosi BR, Karimi M, Jamehshorani $S$ et al. Surveying the effects of an exercise program on the sleep quality of elderly males. Clin Interven Aging [Internet]. 2016;11: 997-1002. Disponible en: https://www. ncbi.nlm.nih.gov/pmc/articles/PMC4968687/pdf/ cia-11-997.pdf

13. Romao LS, Nogueiro AL, Mendes ME, Pinto A, Pimentel $\mathrm{MH}$. Deterioro funcional, miedo a caerse y composición corporal en ancianos institucionalizados. Enferm Clin [Internet]. 2014;25(2):81-86. Doi: 10.1016/j.enfcli.2014.10.005

14. Yáñez $C$, León $P$, Medina $P$. Efecto del tiempo e institucionalización en variables antropométricas apendiculares, en un grupo de adultos mayores independientes y dependientes. Fisioterapia [Internet]. 2015;38(2):60-70. Doi: 10.1016/j. ft.2015.05.001

15. Pinzón S, Garrido F, Reyes V, Lima JS, Raposo MF, Martínez $\mathrm{M}$ et al. Factores predictores de la institucionalización de personas mayores en situación de dependencia en Andalucía. Enferm Clín [Internet]. 2016;26(1):23-30. Doi: 10.1016/j.enfcli.2015.08.003

16. Varela LF, Tello T, Ortiz PJ, Chávez H. Valoración de la higiene del sueño mediante una escala modificada en adultos mayores. Acta Med Peruana [Internet]. 2010;27(4):233-237. Disponible en: http://www.scielo.org.pe/pdf/amp/v27n4/ a05v27n4.pdf

17. Martínez MA. ¿Cuánto hay que dormir para 
un sueño saludable? Rev Neurol [Internet]. 2016;63(S2):S7-S9. Disponible en: http://ses. org.es/docs/rev-neurologia2016.pdf

18. Tlatoa HM, Ocaña HL, Márquez ML, Bermeo J, Gallo AF. El género, un factor determinante en el riesgo de somnolencia. Med Investig [Internet]. 2015;3(1):17-21. Disponible en: http://ri.uaemex. $\mathrm{mx} /$ oca/bitstream/20.500.11799/32807/1/353v03 n01a90413431pdf003.pdf

19. Merino M. Los hábitos de sueño de los españoles. Rev Neurol [Internet] 2016;63(S2):S3-S9. Disponible en: http://ses.org.es/docs/rev-neurologia2016.pdf

20. Medina JH, Fuentes SA, Gil IB, Adam L, Solís F, Sánchez $L$ et al. Guía de práctica clínica, diagnóstico y tratamiento del insomnio en el adulto mayor. Rev Med Inst Mex Seguro Soc [Internet]. 2014;52(1):108-119. Disponible en: http://www. medigraphic.com/pdfs/imss/im-2014/im141v.pdf

21. Madrid JA. Importancia de los horarios del sueño. Rev Neurol [Internet]. 2016;63(2):S10-S11. Disponible en: http://ses.org.es/docs/rev-neurologia2016.pdf

22. Tello T, Alarcón RD, Vizcarra D. Salud mental en el adulto mayor: Trastornos neurocognitivos mayores afectivos y del sueño. Rev Perú Med Exp Salud Publica [Internet]. 2016;33(2):342350. Disponible en: https://www.scielosp.org/article/rpmesp/2016.v33n2/342-350/

23. López F, Fernández $O$, Mareque MA, Fernández L. Abordaje terapéutico del insomnio. Semergen [Internet]. 2012;38(4):233-240. Disponible en: http://www.centropsicologicomca.es/articulos/ ctl_servlet-insomnio.pdf

24. Asociación Americana de Psiquiatría. Trastornos del sueño-vigilia. En: Arlington VA. Guía de consulta de los criterios diagnósticos del DSM-5. $5 a$ ed. London: American Psychiatric Publishing; 2014. 203-224.

25. Ganado E, Garay I, Hernando A, Vega L. Insom- nio. El farmacéutico [Internet]. 2015;(528):33-42. Disponible en: goo.gl/BgizLi

26. Flores A, Alvarado A. Apnea obstructiva del sueño: experiencia en el Hospital San Juan de Dios. Acta Med Costarric [Internet]. 2014;56(1):12-16. Disponible en: www.actamedica.medicos.cr/index.php/Acta_Medica/article/download/825/746

27. Fernández AM. Trastornos del sueño. Medicine [Internet]. 2007;9(86):5550-5557. Doi: 10.1016/ S0211-3449(07)74695-7

28. Fuentes SA, Gil IB, Sánchez F, Adam L, Sánchez LY, Solís F. Diagnóstico y tratamiento del insomnio en el anciano. Guía de práctica clínica. 2011; 20-22.

29. Rodríguez Lozano R, Gómez Conesa A. El fisioterapeuta es el profesional prescriptor del ejercicio físico con fines terapéuticos. Fisioterapia. 2016;38(6):269-270. Doi: 10.1016/j. ft.2016.10.001

30. Cordero A, Masiá MD, Galve E. Ejercicio físico y salud. Rev Esp Cardiol [Internet]. 2014;67(9):748753. Disponible en: http://www.revespcardiol.org/ es/pdf/90341409/S300/

31. Landinez NS, Contreras K, Castro A. Proceso de envejecimiento, ejercicio y fisioterapia. Rev Cub Salud Pub [Internet]. 2012;38(4):562-580. Disponible en: http://www.medigraphic.com/pdfs/ revcubsalpub/csp-2012/csp124h.pdf

32. Vidarte JA, Quintero MV, Herazo Y. Efectos del ejercicio en la condición física funcional y la estabilidad en adultos mayores. Hacia la Promoción de la Salud [Internet]. 2012;17(2):7990. Disponible en: http://www.redalyc.org/ pdf/3091/309126826006.pdf

33. Saz P, Gálvez JJ, Ortiz M, Saz S. Ejercicio físico. Med Nat [Internet]. 2011;5(1):18-23. Disponible en: https://dialnet.unirioja.es/descarga/articulo/3401250.pdf

34. Jurado J, Jiménez A, Ordoñez I, Roldán B, Chi- 
ca $M$, Rueda $M$ et al. Efecto del ejercicio sobre la somnolencia y la calidad de vida en pacientes con apneas del sueño sin indicación de tratamiento con CPAP. Rev Esp Patol Tocac [Internet]. 2015;27(4):201-271. Disponible en: https:// www.neumosur.net/files/publicaciones/Revistas/2015/2_original-rev2015-v27-n4.pdf

35. Gallego J, Aguilar JM, Cangas AJ, Lorenzo JJ, Franco C, Mañas I. Programa de natación adaptada para personas mayores dependientes: beneficios psicológicos, físicos, y fisiológicos. Rev Psicol Dep [Internet]. 2012;21(1):125133. Disponible en: https://ddd.uab.cat/pub/ revpsidep/revpsidep_a2012v21n1/revpsidep_ a2012v21n1p125.pdf 